\title{
Surface Modification of Ni-Cr-Mo Steel by Multifunction
}

\section{Cavitation}

\author{
Masataka Ijiri, Daichi Shimonishi, Daisuke Nakagawa, Kumiko Tanaka and Toshihiko Yoshimura \\ Tokyo University of Science, Yamaguchi, 1-1-1 Daigaku-Dori, Sanyo-Onoda, Yamaguchi 756-0084, Japan
}

\begin{abstract}
The multifunction cavitation (MFC) technique is referred to as a mechanical-electrochemical cavitation method because it includes both mechanical action by microjets (MJs), with high pressure from water jet cavitation (WJC), and electrochemical action by MJs, with the high-temperature reaction field of ultrasonic cavitation (UC). Recently, when MFC was applied to Cr-Mo (JIS-SCM435) steel, the corrosion resistance and compressive residual stress in rust were improved, and voids and cracks of the specimen interior were suppressed compared to that with WJC. It is of interest whether this phenomenon occurs similarly in other low-alloy steels processed by MFC. In the present study, MFC was applied to the modification of Ni-Cr-Mo steel (JIS-SNCM630) surfaces. SNCM630 processed by MFC exhibited improved corrosion resistance and compressive residual stress in rust, and voids and cracks in the interior were not generated from the specimen surface to any significant extent.
\end{abstract}

Key words: Water jet cavitation, multifunction cavitation, SNCM630 steel, Ni-Cr-Mo steel, surface reforming, dissolved oxygen.

\section{Introduction}

Low alloy steel is a steel material with improved corrosion resistance by alloying elements of several mass percent or less, or of 1 mass $\%$ or less in various combinations based on carbon steel. It is slightly more expensive than carbon steel, but has higher corrosion resistance against specific corrosion. Improvement of the fatigue characteristics and corrosion resistance is not always satisfactory with the conventional surface modification techniques, because the environments in which these materials are used, are becoming increasingly harsh.

Surface modification technologies such as WJC (water jet cavitation) have thus been applied to improve the stress corrosion cracking [1] and fatigue strength [2] of alloy steels. In the WJC process, high pressure occurs when a cavitation caused by high pressure water jetted from a nozzle collapses on the metal surface. This impact pressure results in slight plastic deformation of the surface layer, and generates compressive residual stress by an elastic restraining

Corresponding author: Masataka Ijiri, Post-doctoral researcher, research fields: Ti alloy, water jet cavitation. force from the lower layer portion and the surroundings. When the compression force is converted to compression deformation, the deformation returns to its original state after the cavitation collapse; however, if a small amount of plastic deformation occurs, then compressive residual stress is generated after cavitation collapse. We have recently reported that voids and cracks tend to form inside specimens in exchange for a high compressive residual stress with an increase in pressure applied to the specimen surface [3].

We have developed a new WJC technology [4] that is both a mechanical and electrochemical cavitation method because it has electrochemical action from the high-temperature reaction field of UC (ultrasonication) in addition to mechanical action from WJC by high-pressure MJs (microjets). This technique is termed MFC (multifunction cavitation) because it combines new functions when processing the surface of various materials. UC generates both low- and high-pressure regions around small bubbles (several micrometers in size) by the application of strong ultrasonic waves in a liquid. Bubbles with a high-temperature reaction field are generated by 
repeated isothermal expansion and adiabatic compression. However, in the case of UC (and in contrast to WJC processing), these bubbles are very small and the collapse pressure is low, such that the pressure applied to the specimen surface and the emitted shock waves is weak. As a result, almost no processing is applied to the specimen surface. MFC generates air bubbles that have a high-temperature reaction field by UC in air bubbles generated by WJC. It is possible to process the specimen surface in a similar way to that with WJC. Various material properties have been extracted to date using this technology. For example, it has been reported that MFC-processed Cr-Mo steel (SCM435) has improved corrosion resistance in rust, and voids and cracks are not formed to any significant extent in the specimen interior [5]. This phenomenon also indicates that the specimen surface was heated to high-temperature in water. It is of interest whether this phenomenon occurs similarly in other low-alloy steels processed by MFC. In the present study, MFC was applied to the modification of Ni-Cr-Mo steel (SNCM630) surfaces, and the dependence of the microstructure and hardness on MFC was investigated and the results compared to those for WJC.

\section{Experimental Methods}

The material used for these tests was Ni-Cr-Mo steel (SNCM630), a structural machine steel, the chemical composition of which is shown in Table 1. Round bar (rod) specimens were heated at $870{ }^{\circ} \mathrm{C}$ as a solution treatment, followed by quenching. Tempering was performed at $580{ }^{\circ} \mathrm{C}$. The specimens were subsequently cut into rectangular specimens with dimensions of $100 \times 100 \times 3 \mathrm{~mm}^{3}$. Fig. 1 shows a schematic diagram of the equipment used for $\mathrm{MFC}$ processing. In conventional WJC apparatus, a nozzle is fixed in water at room temperature and water jets are pumped at a discharge pressure of $35 \mathrm{MPa}$. The nozzle diameter used in the present work was $0.8 \mathrm{~mm}$. The distance between the nozzle and the specimen was set to be $65 \mathrm{~mm}$. In MFC treatment, an ultrasonic transducer is set to the vertical direction of the water jet nozzle for conventional WJC, and ultrasonic waves are sonicated to the water jet flow from the water jet nozzle. During the present study, UC was performed at $225 \mathrm{~W}$ and $28 \mathrm{kHz}$. Residual stress measurement, hardness test, optical microscope observation, scanning electron microscope were measured under the same conditions as previously reported [5]. For the measurement of the surface potential, the function of a KFM (Kelvin probe force microscopy) provided in a multi-compatible miniature probe microscope (AFM5200S, Hitachi High-Tech Sciences Co., Ltd.) was used. The measurement area was $100 \times 100 \mu \mathrm{m}^{2}$, and the value obtained by subtracting three lines by line analysis was averaged. In preparation for observations, specimens were corroded in 5 vol.\% Nital etchant solutions. Prior to all post-processing characterization procedures, specimens were cut into 1 $\times 1 \mathrm{~cm}^{2}$ test samples. The DO (dissolved oxygen) values of water, in which cavitation had occurred in an SUS310S reactor for $30 \mathrm{~min}$, were obtained using a portable analyzer (OM-71, Horiba, Ltd.).

Table 1 Chemical composition of the SNCM630.

\begin{tabular}{lllllllll}
\hline $\mathrm{C}$ & $\mathrm{Si}$ & $\mathrm{Mn}$ & $\mathrm{P}$ & $\mathrm{Ni}$ & $\mathrm{Cr}$ & $\mathrm{Mo}$ & $\mathrm{Cu}$ & $\mathrm{Fe}$ \\
\hline 0.29 & 0.24 & 0.44 & 0.11 & 2.93 & 3.01 & 0.56 & 0.03 & Bal. \\
\hline
\end{tabular}

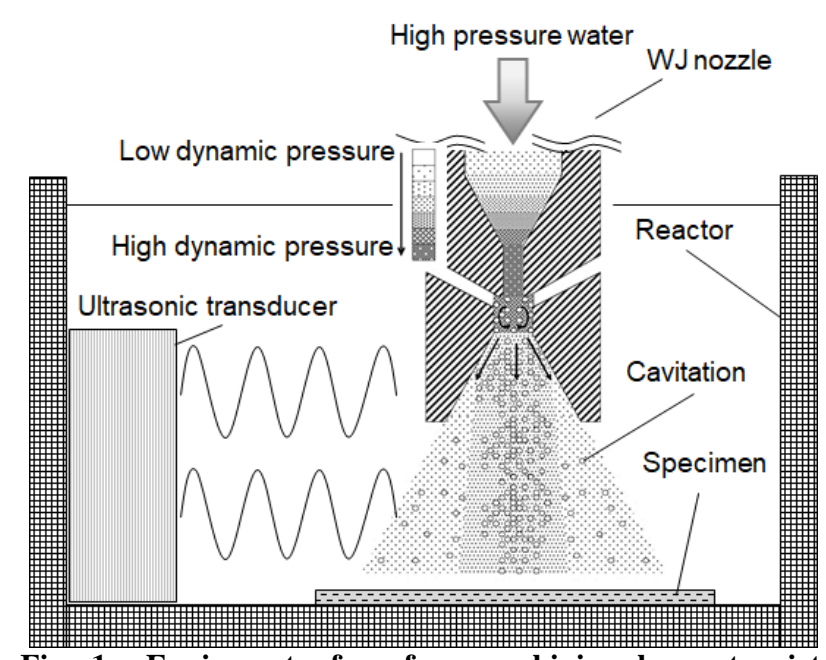

Fig. 1 Equipment of surface machining by water jet cavitation under ultrasonic transducer. 


\section{Results and Discussion}

\subsection{Improvements in the Corrosion Resistance of the} Specimen Surface

Fig. 2 shows surface photographs of specimens processed with (a) WJC and (b) MFC. The specimen surface was painted with an oil-based magic ink prior to processing to identify the peening position. Observation of the oil-based magic ink marking after processing indicated the diameter of the WJC processed region was $41.3 \mathrm{~mm}$, while that with MFC processing was $42.7 \mathrm{~mm}$; therefore, the MFC processing area was slightly larger than the WJC processing area. In addition, when the processed specimen was kept at room temperature for several months, corrosion proceeded after WJC processing, where there was almost no corrosion after MFC processing. In our previous report [5], it was considered that a dense oxide film was formed because the surface temperature increased in the MFC-processed SCM435. Therefore, it is considered that corrosion of the SNCM630 steel was prevented due to the formation of a dense oxide film on the specimen by the increase of temperature at the surface.

Fig. 3 shows SEM micrographs of the specimen side after WJC and MFC treatment. The particle size in the depth direction from the surface after each process was not significantly affected. In the near-surface region, no significant corrosion was observed after WJC processing, whereas corrosion occurred easily after MFC processing.

Thus, selective oxidation is considered to have occurred in the bulk of the processed specimen. The element that is difficult to oxidize in this specimen is $\mathrm{Cr}$, which migrates to the topmost surface and forms a $\mathrm{Cr}$-poor region beneath the surface during heating by MFC processing. As a result, rust can be easily generated because the amount of $\mathrm{Cr}$ is decreased in the near-surface region.

The corrosion resistance of the specimen surfaces was assessed by measuring the surface potentials, and the results are summarized in Table 2 .
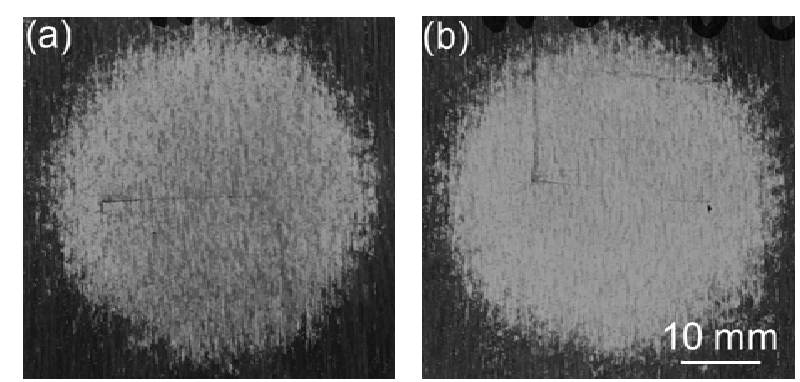

Fig. 2 Change in surface morphology by (a) WJC and (b) MFC treatment.
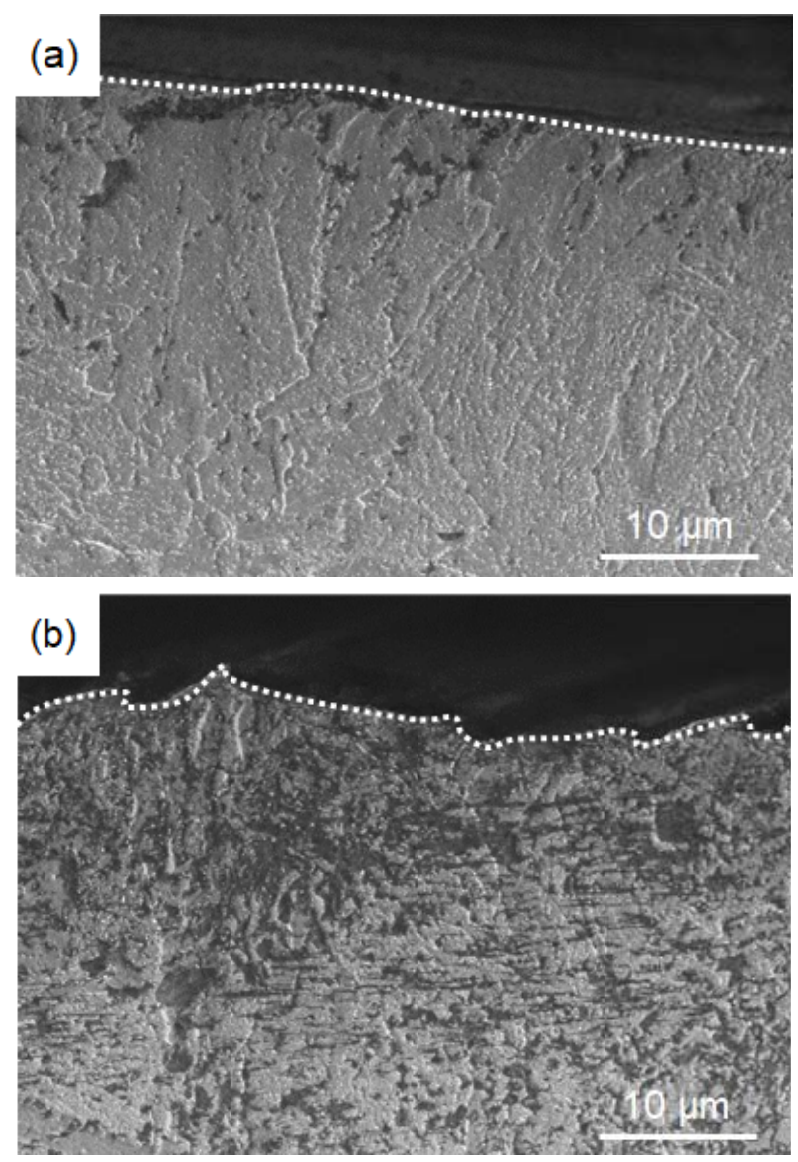

Fig. 3 SEM images of the specimen side after (a) WJC treatment and (b) MFC treatment. The dashed line indicates the surface of specimen.

Table 2 Surface potential of SNCM630 after various treatments.

\begin{tabular}{ll}
\hline & Surface potential \\
\hline Mirror-finished specimen & $359 \pm 25 \mathrm{mV}$ \\
UC & $342 \pm 18 \mathrm{mV}$ \\
WJC & $329 \pm 18 \mathrm{mV}$ \\
MFC & $445 \pm 16 \mathrm{mV}$ \\
\hline
\end{tabular}


The surface potential of the specimen surface processed using UC and WJC was lower than those of the other processed specimen surfaces, and also lower than the original material. The work function corresponding to this surface potential decreases as the surface roughness increases. During UC, the thermodynamic stability of the bubbles decreases after repeated isothermal expansion and adiabatic compression, which leads to bubble collapse.

The internal temperature of the bubble is high but the bubble size is small, so that the amount of heat transferred to the specimen surface is minimal. As a result, and because the collapse pressure of each bubble is also small, there are no significant surface modifications due to heating and pressure. During WJC, the collapse of cavitation bubbles imparts high pressure to the surface and so defects are increased due to a slight surface temperature increase and mechanical action. This could deteriorate the corrosion resistance of the specimen surface. In the case of the MFC-processed specimen, which exhibited the highest corrosion resistance, it is likely that the hot spots inside the bubbles significantly increased the sample surface temperature upon collapse of the cavitation bubbles, such that a passive layer was formed and the corrosion resistance was improved.

The reason for the selective oxidation inside the specimen during MFC processing was investigated by monitoring the water temperature, and Fig. 4 summarizes the results. While UC did not increase the water temperature, both WJC and MFC did raise the temperature in the reactor. This occurred as a result of the pressurization of the water by the high-pressure pump because the kinetic energy of the water was converted to thermal energy. From the viewpoint of bubble energy, it is considered that both WJC and MFC generate larger bubble collapse energies than UC processing, which also results in an increase in water temperature. The slightly higher temperature obtained during MFC than during WJC processing is due to the application of ultrasonic energy, which reflects the hot spots in the bubbles generated by MFC.

Fig. 5 plots the DO in the reactor as a function of processing time for each processing method. The DO concentration was almost unchanged during UC, whereas it decreased during both WJC and MFC processing. In WJC processing, the pressure applied to the specimen surface increases due to the shock wave generated by the MJs. A part of the foam is deformed or the bubbles become large again over a very short time span, which results in an increase of both the water temperature and the temperature of the processed surface. As a result, it is considered that DO combines with $\mathrm{Cr}$ to form oxides on the processed specimen surface, and thus the DO decreases.

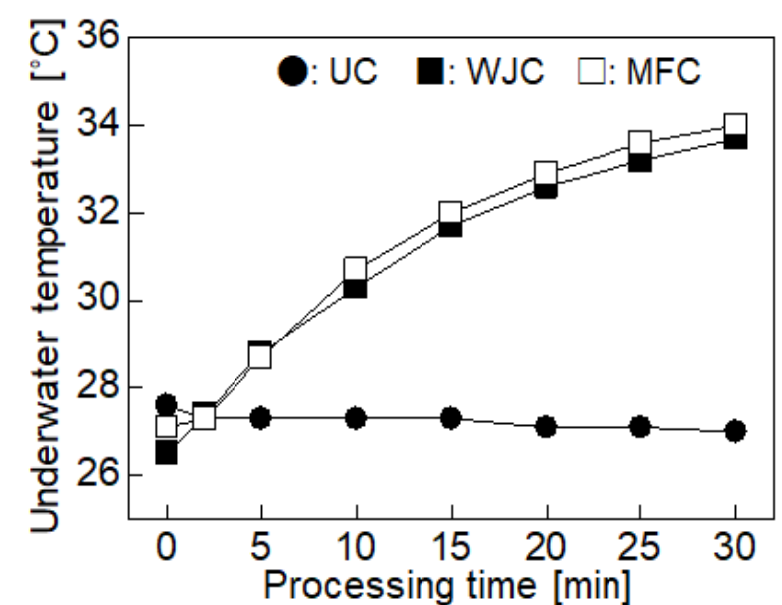

Fig. 4 Underwater temperature as a function of the processing time.

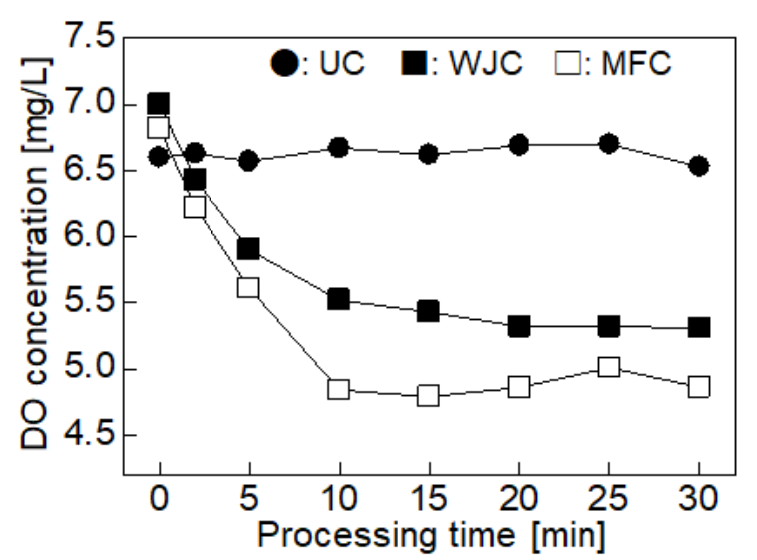

Fig. 5 DO concentration as a function of the processing time. 
During MFC, the bubbles (which have a high internal temperature and pressure) increase the sample surface temperature to a greater extent than during WJC processing due to the hot spot phenomenon inside the bubbles. Thus, the DO concentration is reduced to a greater degree as a passive layer is generated.

\subsection{Influence of Residual Stress from Specimen Surface to Interior}

Table 3 shows the results of residual stress measurements. To further increase the compressive residual stress generated after $\mathrm{WJC}, \mathrm{UC}$ and $\mathrm{MFC}$ processing, the specimen surface was first subjecting to hard grinding, which imparted tensile residual stress. Compared to that without tensile residual stress applied, the compressive residual stress is considered to increase after processing by a shakedown effect. These measurements were conducted in a state without rust on the specimen surface just after each processing, and were performed from the grinding direction in the as-received specimen (parallel direction). It was confirmed that the tensile residual stress was imparted in the grinding direction of the WJC, UC and MFC unprocessed specimens, while the compressive residual stress was imparted in the vertical direction. In UC (and in contrast to WJC and MFC processing) where the improvement of residual stress was the least, the bubbles are very small and the collapse pressure is low, such that the pressure applied to the specimen surface and the emitted shock waves are weak.

As a result, almost no compressive residual stress is applied to the specimen surface. On the other hand, in WJC and MFC processing, compressive residual stress is generated when the specimen surface is compressed by the pressure from cavitation collapse because the MJ applies force in the lateral direction, which leads to plastic deformation around the region that is elastically constrained by its surroundings. Fig. 6 shows the change in the micro Vickers hardness for each processed surface in the depth direction, where the oxide film and peening marks were removed prior to measurement. The hardness of the near-surface region in the unprocessed specimen was $256 \mathrm{HV}$. The hardness distribution of the unprocessed specimen remained unchanged in the depth direction. It is considered that the unprocessed specimen is not significantly influenced by the strong surface grinding that was performed to impart tensile residual stress. The hardness in the near-surface region of the WJC-processed specimen was $270 \mathrm{HV}$ and decreased at a depth of ca. $0.75 \mathrm{~mm}$ from the surface layer. On the other hand, the hardness in the near-surface region of the MFC-processed specimen was $264 \mathrm{HV}$. The hardness decreased at a depth of ca. $0.5 \mathrm{~mm}$ from the surface layer. This change in hardness is dependent on the value (compressive stress field) where compressive residual stress was added in the depth direction. The compressive stress field is deeper in WJC than in MFC.

It is thus considered that compressive residual stress was alleviated because part of the dislocation obtained

Table 3 Residual stress of SNCM630 after various treatments.

\begin{tabular}{llll}
\hline & & $\begin{array}{l}\text { Half value } \\
\text { breadth method }\end{array}$ & $\begin{array}{l}\text { Peak top } \\
\text { method }\end{array}$ \\
\hline \multirow{2}{*}{ As-received } & Vertical direction & $-238 \mathrm{MPa}$ & $-234 \mathrm{MPa}$ \\
& Parallel direction & $+155 \mathrm{MPa}$ & $+107 \mathrm{MPa}$ \\
\hline UC & $-196 \mathrm{MPa}$ & $-208 \mathrm{MPa}$ \\
WJC & $-450 \mathrm{MPa}$ & $-483 \mathrm{MPa}$ \\
MFC & $-481 \mathrm{MPa}$ & $-489 \mathrm{MPa}$ \\
\hline
\end{tabular}

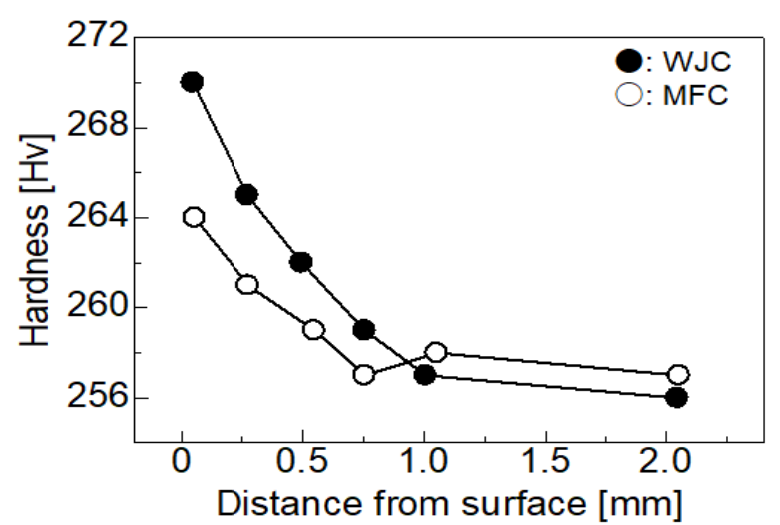

Fig. 6 Hardness change of the specimens after each treatment from surface to interior. 
by work hardening disappeared when the specimen surface was heated by bubbles with hot spots.

Fig. 7 shows SEM images of the specimen side in the vicinity of the surface after (a) WJC and (b) MFC processing. The microstructure of the unprocessed specimen was simple with only granular cementite and ferrite observed. Granular cementite and ferrite were also observed in the various processed specimens. The surface of each processed specimen side is very rough due to the influence of peening. Cementite grains decreased overall in the vicinity of the surface compared with the unprocessed material. Although the grain size was dependent on the observation region, particles of approximately 0.35 $\mu \mathrm{m}$ were observed near the surface.

Fig. 8 shows an SEM image of the specimen side after WJC processing. This observation was performed at a depth of approximately $0.5-1 \mathrm{~mm}$ from the surface. Voids were observed at grain boundaries. They were also observed in the WJC-processed SCM435, and were mainly observed at grain boundaries [3]. No voids or cracks were observed at depths of approximately $2-3 \mathrm{~mm}$ from the surface. WJC processing thus imparts compressive residual stress without changing the properties of the material surface. However, it was revealed that voids are likely to be formed at grain boundaries after WJC processing. The specimen side after MFC processing was observed using SEM; however, no grain boundaries similar to the specimen side after WJC processing were observed.

Fig. 9 shows SEM image of the specimen side after MFC processing.

Cementite grains were observed within the grains of ferrite, and grains with a size exceeding approximately $0.75 \mathrm{~m}$ were present. Relatively small grain sizes of ca. 0.1 to $0.3 \mu \mathrm{m}$ were distributed in other places. It is considered that this variation in grain growth is affected by high-temperature and high-pressure bubbles colliding with the surface by the cavitation jet. The interior of SCM 435 processed by MFC in our previous report changed from pearlite to spheroidized cementite after processing [3]. It is thus considered that
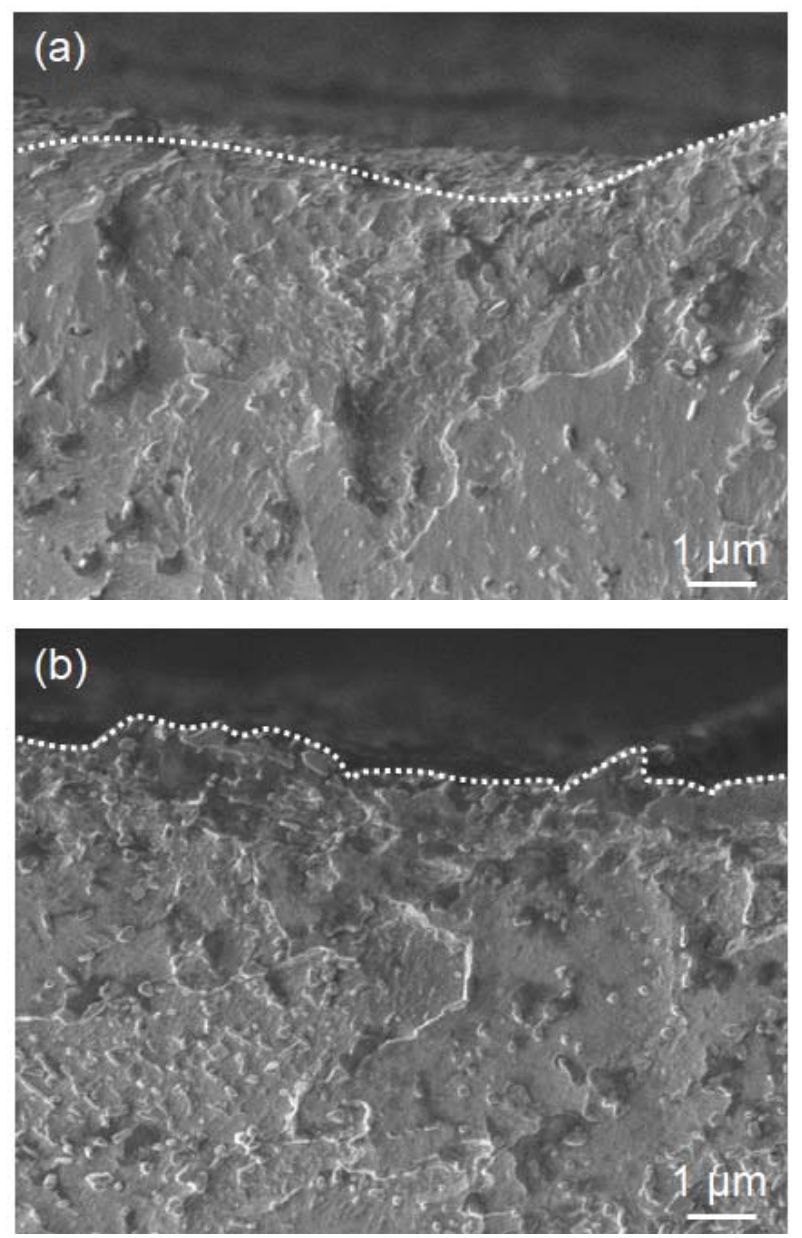

Fig. 7 SEM images of the specimen side after (a) WJC treatment and (b) MFC treatment. The dashed line indicates the surface of specimen.

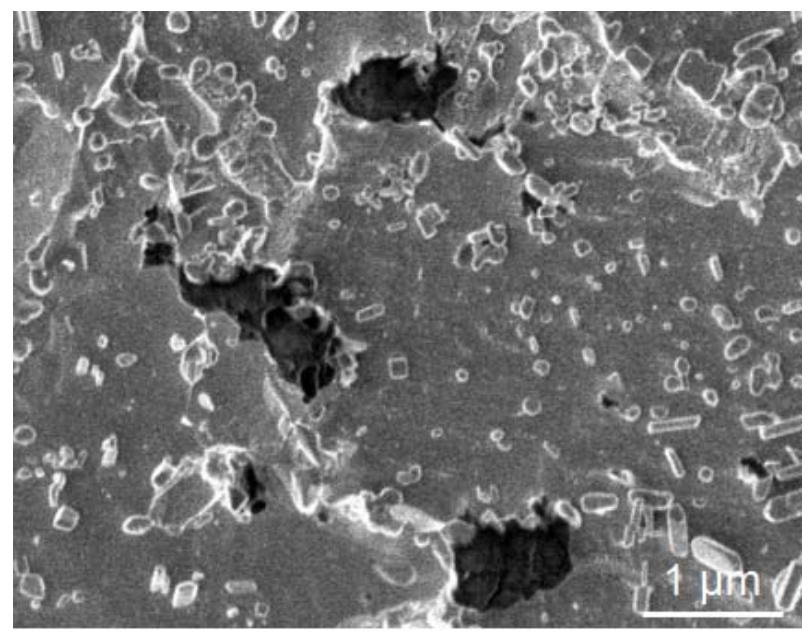

Fig. 8 SEM image of the specimen side after WJC treatment. 


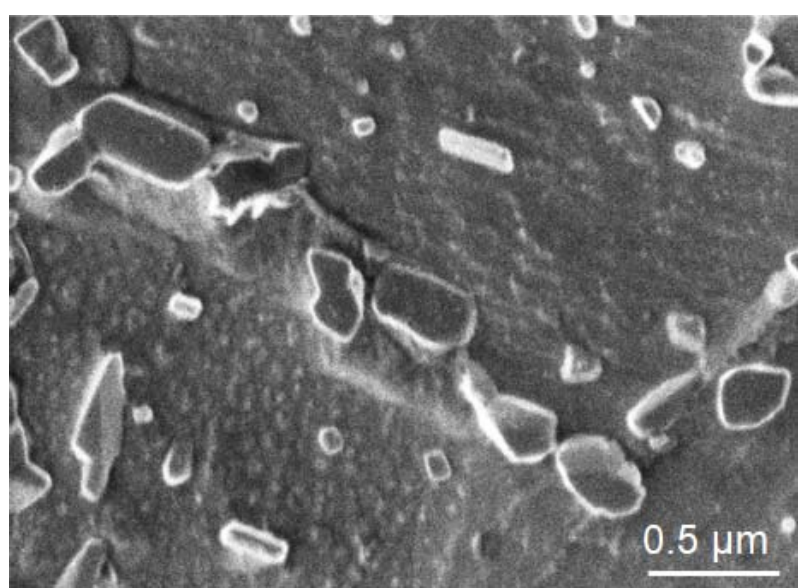

Fig. 9 SEM image of the specimen side after MFC treatment.

the specimen interior was annealed. Not only in SCM 435 but also in SNCM 630, a temperature rise was confirmed on the surface and in the bulk after processing. In the future, it will be necessary to investigate the temperature distribution in the depth direction from the processed surface.

\section{Conclusions}

SNCM630 processed by MFC exhibited improved corrosion resistance in rust and compressive residual stress, and no voids or cracks were generated in the bulk interior from the specimen surface to any significant extent. A phenomenon similar to that reported for SCM435 occurs. As a result, it became clear that the surface modification technique by MFC processing is not only applicable to SCM435, but also a universal phenomenon. In the specimen processed by WJC, it was revealed that voids tended to occur in the depth region of $0.5-1 \mathrm{~mm}$ from the topmost surface. Moreover, no voids or cracks were observed at depths of 2-3 $\mathrm{mm}$ from the surface. Corrosion resistance was improved via the formation of an oxide film by selective oxidation, and by a reduction of surface defects. It is considered that this oxide film was formed by the reaction of DO in the water with $\mathrm{Cr}$ on the metal surface during the MFC treatment.

\section{Acknowledgments}

This work was supported by the Innovative Science and Technology Initiative for Security program of the Acquisition, Technology \& Logistics Agency (ATLA) of Japan.

\section{References}

[1] Gompf, B., Gunther, R., Nick, G., Pecha, R., and Eisenmenger, W. 1997. "Resolving Sonoluminescence Pulse Width with Time-Correlated Single Photon Counting." Phys. Rev. Lett. 79: 1405-8.

[2] Odhiambo, D., and Soyama. H. 2003. "Cavitation Shotless Peening for Improvement of Fatigue Strength of Carbonized Steel.” Inter. J. Fatigue 25: 1217-22.

[3] Ijiri, M., Shimonishi, D., Nakagawa, D., and Yoshimura, T. 2017. "Evolution of Microstructure from the Surface to the Interior of Cr-Mo Steel by Water Jet Peening." Materials Sciences and Applications 8: 708-15.

[4] Yoshimura, T., Tanaka, K., and Yoshinaga, N. 2016. "Development of Mechanical-Electrochemical Cavitation Technology.” J. Jet Flow Eng. 32: 10-7.

[5] Ijiri, M., and Yoshimura, T. 2018. "Evolution of Surface to Interior Microstructure of SCM435 Steel after Ultra-High-Temperature and Ultra-High-Pressure Cavitation Processing.” J. Mater. Process. Technol. 251: 160-7. 